\title{
Clinical Extrapolation of the Effects of Dolutegravir and Other HIV Integrase Inhibitors on Folate Transport Pathways ${ }^{\text {[ }}$
}

\author{
Maciej J. Zamek-Gliszczynski, ${ }^{1}$ Xuexiang Zhang, ${ }^{1}$ Jennypher Mudunuru, Yewei Du, Jian-Lu Chen, \\ Kunal S. Taskar, Jane Huang, Yong Huang, and Elizabeth H. Romach \\ GlaxoSmithKline, Collegeville, Pennsylvania (M.J.Z.-G., J.M.); GlaxoSmithKline, Ware, United Kingdom (K.S.T.); Bio/VT, Santa Clara, \\ California (X.Z., Y.D., J.-L.C., J.H., Y.H.); and ViiV Healthcare, Research Triangle, North Carolina (E.H.R.)
}

Received April 18, 2019; accepted June 4, 2019

\begin{abstract}
Preliminary analysis of ongoing birth surveillance study identified evidence of potential increased risk for neural tube defects (NTDs) in newborns associated with exposure to dolutegravir at the time of conception. Folate deficiency is a common cause of NTDs. Dolutegravir and other HIV integrase inhibitor drugs were evaluated in vitro for inhibition of folate transport pathways: proton-coupled folate transporter (PCFT), reduced folate carrier (RFC), and folate receptor $\alpha$ (FR $\alpha)$ mediated endocytosis. Inhibition of folate transport was extrapolated to the clinic by using established approaches for transporters in intestine, distribution tissues, and basolateral and apical membranes of renal proximal tubules (2017 FDA Guidance). The positive controls, methotrexate and pemetrexed, demonstrated clinically relevant inhibition of PCFT, RFC, and FR $\alpha$ in folate absorption, distribution, and renal sparing. Valproic acid was used as a negative control that elicits folateindependent NTDs; valproic acid did not inhibit PCFT, RFC, or FR $\alpha$. At clinical doses and exposures, the observed in vitro inhibition of FR $\alpha$ by dolutegravir and cabotegravir was not flagged as clinically relevant; PCFT and RFC inhibition was not observed in vitro. Bictegravir inhibited both PCFT and FR $\alpha$, but the observed inhibition did not reach the criteria for clinical relevance. Elvitegravir and raltegravir inhibited PCFT, but only raltegravir inhibition of intestinal PCFT was flagged as
\end{abstract}

potentially clinically relevant at the highest $1.2-\mathrm{g}$ dose (not the $400-\mathrm{mg}$ dose). These studies showed that dolutegravir is not a clinical inhibitor of folate transport pathways, and it is not predicted to elicit clinical decreases in maternal and fetal folate levels. Clinically relevant HIV integrase inhibitor drug class effect on folate transport pathways was not observed.

\section{SIGNIFICANCE STATEMENT}

Preliminary analysis of ongoing birth surveillance study identified evidence of potential increased risk for neural tube defects (NTDs) in newborns associated with exposure to the HIV integrase inhibitor dolutegravir at the time of conception; folate deficiency is a common cause of NTDs. Dolutegravir and other HIV integrase inhibitor drugs were evaluated in vitro for inhibition of the major folate transport pathways: proton-coupled folate transporter, reduced folate carrier, and folate receptor $\alpha$-mediated endocytosis. The present studies showed that dolutegravir is not a clinical inhibitor of folate transport pathways, and it is not predicted to elicit clinical decreases in maternal and fetal folate levels. Furthermore, clinically relevant HIV integrase inhibitor drug class effect on folate transport pathways was not observed.

\section{Introduction}

Dolutegravir is an integrase inhibitor for the treatment of HIV, a drug class that also includes bictegravir, elvitegravir, raltegravir, and cabotegravir (Han et al., 2017). Preliminary analysis of an ongoing birth surveillance study identified evidence of a potential increased risk for neural tube defects (NTDs) in newborns associated with exposure to dolutegravir at the time of conception (Zash et al., 2018). This observation was unexpected because standard preclinical reproductive toxicity studies in rats and rabbits did not identify fetal abnormalities at exposures up to 27 -fold higher than at the maximum indicated human dose (2018).

Folate is critical to proper neural tube embryonic development in the first 4 weeks of human pregnancy (Botto et al., 1999). Women of

${ }^{1}$ M.J.Z.-G. and X.Z. contributed equally to this work.

This study was funded by ViiV Healthcare.

https://doi.org/10.1124/dmd.119.087635.

S This article has supplemental material available at dmd.aspetjournals.org. childbearing potential are therefore encouraged to supplement folic acid (Botto et al., 1999; Kancherla et al., 2018). Since neural tube development occurs before most women know they are pregnant, many countries have implemented folic acid fortification of food via wheat and/or maize flour supplementation (Kancherla et al., 2018). Likewise, rodent chow of laboratory animals is supplemented with folic acid, as these animals have no access to natural vitamin sources. Notably, the food supply is not fortified with folic acid in Botswana, where the dolutegravir birth surveillance study was conducted (Kancherla et al., 2018; Zash et al., 2018).

Drug-induced folate deficiency leading to increased incidence of adverse effects, including neurotoxicity and embryonic NTDs, as well as rescue/prevention with folic acid supplementation, are well established for the antifolate drug methotrexate (DeSesso and Goeringer, 1991; Cohen, 2017). Likewise, the intravenous antifolate pemetrexed is indicated for use only with folic acid premedication and oversupplementation to avoid serious toxicities associated with folate depletion in cancer patients (2019). Notably, folate deficiency is not the sole cause of increased NTD risk associated with drug treatment. For example, the

ABBREVIATIONS: DHFR, dihydrofolate reductase; FR $\alpha$, folate receptor $\alpha$; IC, inhibitory concentration; MDCK-II, Madin-Darby canine kidney-II; NTDs, neural tube defects; PCFT, proton-coupled folate transporter; RFC, reduced folate carrier. 


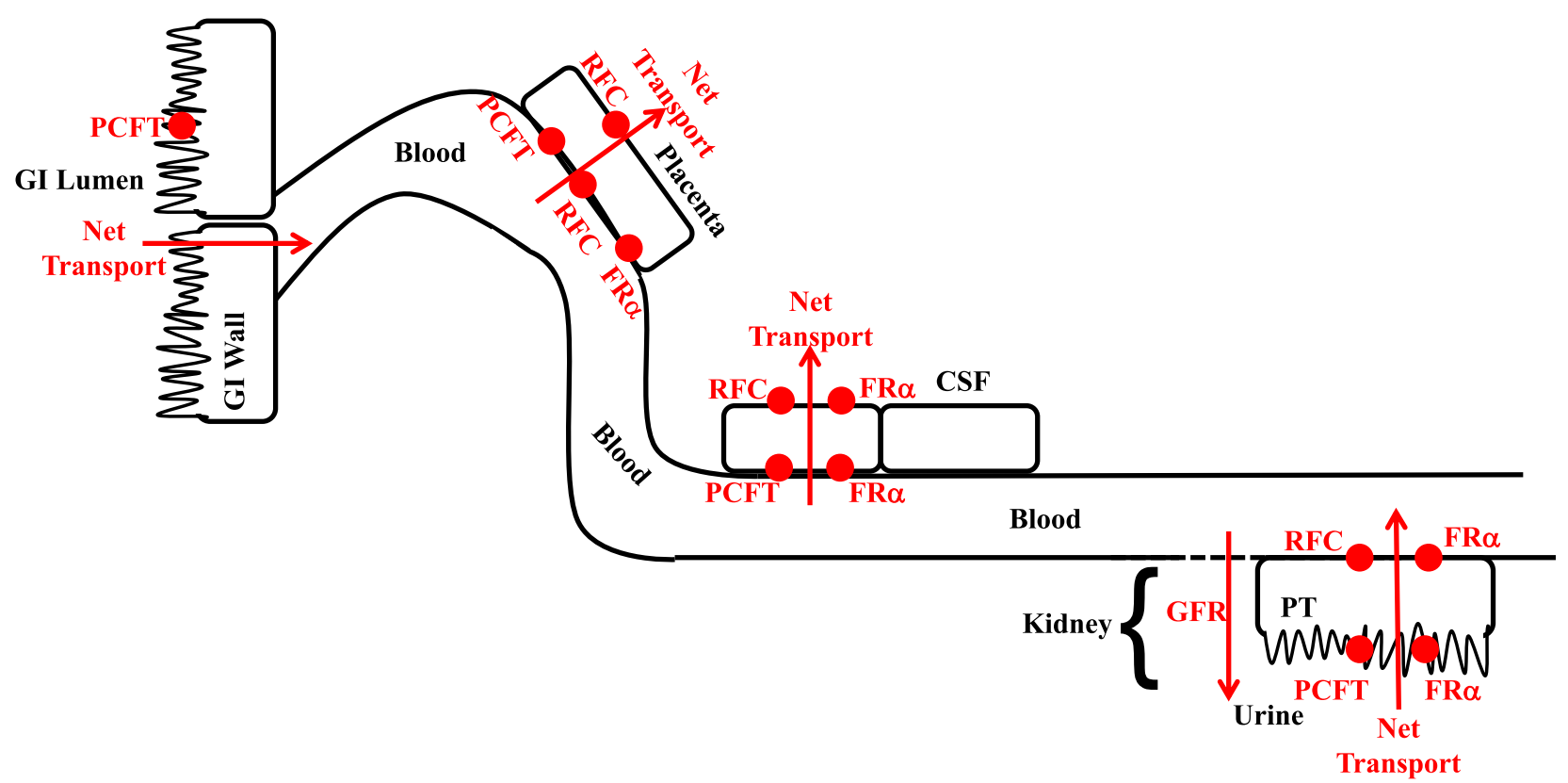

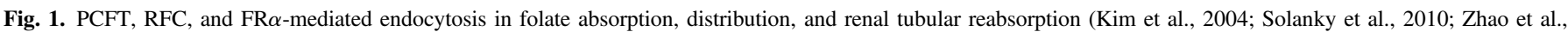
2011). CSF, cerebrospinal fluid; GFR, glomerular filtration rate; PT, renal proximal tubule.

antiepileptic drug valproic acid increases the incidence of NTDs 4-fold relative to other antiepileptic therapies used in pregnant women (2011). As folic acid supplementation does not reduce the incidence of valproic acid NTDs, this drug is considered to elicit NTDs via unknown folateindependent mechanism(s) (Hansen et al., 1995; Craig et al., 1999; Candito et al., 2007).

Folate's hydrophilic nature results in negligible passive membrane permeability and transport-mediated disposition. Transport mechanisms governing folate intestinal absorption, distribution, and renal sparing are depicted in Fig. 1 (Kim et al., 2004; Solanky et al., 2010; Zhao et al., 2011). The proton-coupled folate transporter (PCFT), a solute carrier family transporter, is primarily responsible for folic acid intestinal absorption. Distribution of folate into the tissues, including the placenta and brain, involves the solute carriers PCFT and reduced folate carrier (RFC), as well as folate receptor $\alpha(\mathrm{FR} \alpha)$-mediated endocytosis. Like other hydrophilic nutrients and vitamins, folate is not bound to plasma protein in blood and therefore undergoes extensive glomerular filtration in the kidney. Since folate passive tubular reabsorption is negligible owing to low permeability, folate requires efficient tubular reabsorption (a.k.a., renal sparing), so that blood folate is not rapidly excreted in urine (Zhao et al., 2011). All three folate transport processes are involved in folate active tubular reabsorption.

Another mechanism of folate deficiency is inhibition of dihydrofolate reductase (DHFR), which converts dietary folic acid into reduced folates used for nucleic and amino acid synthesis. Dolutegravir is not an inhibitor of DHFR (Cabrera et al., 2019), and since this mechanism has already been ruled out, the present study focused on folate transport.

HIV integrase inhibitors were evaluated for inhibition of PCFT folic acid transport, RFC transport of reduced folate, and FR $\alpha$-mediated folic acid endocytosis. Inhibition of these folate transport pathways has been investigated in vitro and was extrapolated to the clinic by using established approaches based on regulatory recommendations for drug-drug interactions for transport processes in intestine, distribution tissues, and basolateral and apical membranes of the renal proximal tubules (FDA, 2017). The aim of this work was to determine whether dolutegravir is a clinically relevant inhibitor of the three major folate transport pathways, which could increase the risk of maternal and fetal folate deficiency and NTDs, as well as to investigate potential HIV integrase inhibitor drug class effect on folate transport.

\section{Materials and Methods}

\section{Materials}

Dolutegravir and cabotegravir were provided by GlaxoSmithKline (Zebulon, NC). Bictegravir was purchased from Medkoo Biosciences (Morrisville, NC). Raltegravir and elvitegravir were obtained from Cayman Chemical (Ann Arbor, MI). Methotrexate, pemetrexed, valproic acid, and bromosulfophthalein were purchased from Sigma-Aldrich (St. Louis, MO). $\left.{ }^{3} \mathrm{H}\right]$-folic acid and $\left[{ }^{3} \mathrm{H}\right]$-methotrexate were purchased from Moravek (Brea, CA). All other chemicals were of reagent grade and are readily available from commercial sources.

\section{Folate Transport Assays}

The test system was Madin-Darby canine kidney-II (MDCK-II) cell polarized monolayers grown on permeable supports of 96-well transwell plates. The MDCK-II cells were individually transfected to express PCFT, RFC, FR $\alpha$, or vector control transiently.

Assay conditions for PCFT, RFC, and FR $\alpha$ are summarized in Fig. 2. PCFT is expressed on the apical membrane in the kidney/MDCK-II renal cell line (Zhao et al., 2011); hence, all incubations in this study were done on the apical side. The test article was preincubated for 30 minutes at $\mathrm{pH} 7.4$ on the apical side to account for potential time-dependent inhibition and to allow the test article to equilibrate with cells, followed by a 5-minute coincubation of $10 \mathrm{nM}$ $\left[{ }^{3} \mathrm{H}\right]$-folic acid with the test article at $\mathrm{pH} 5.5$ (PCFT cotransports folic acid with a proton). Folic acid uptake in PCFT versus vector control cells was determined. Samples for test article concentration and lactate dehydrogenase were collected from the apical side.

RFC is localized on the basolateral membrane of kidney proximal tubules/ MDCK-II renal cell line (Zhao et al., 2011); thus, all test article incubations were done on the basolateral side (see Fig. 2B). RFC is the reduced folate carrier and does not transport folic acid, but its reduced form; $0.5 \mu \mathrm{M}\left[{ }^{3} \mathrm{H}\right]$-methotrexate, was used as a reduced form of folate in these studies (Milstein et al., 2001). The 30minute and 5-minute coincubations were similar to that of PCFT, except the $\mathrm{pH}$ of the medium for RFC experiments was 7.4 during coincubation (RFC activity is optimal at neutral pH) (Matherly et al., 2007; Zhao et al., 2011). Methotrexate 
SCHEMATIC OF PCFT TRANSPORT EXPERIMENTS

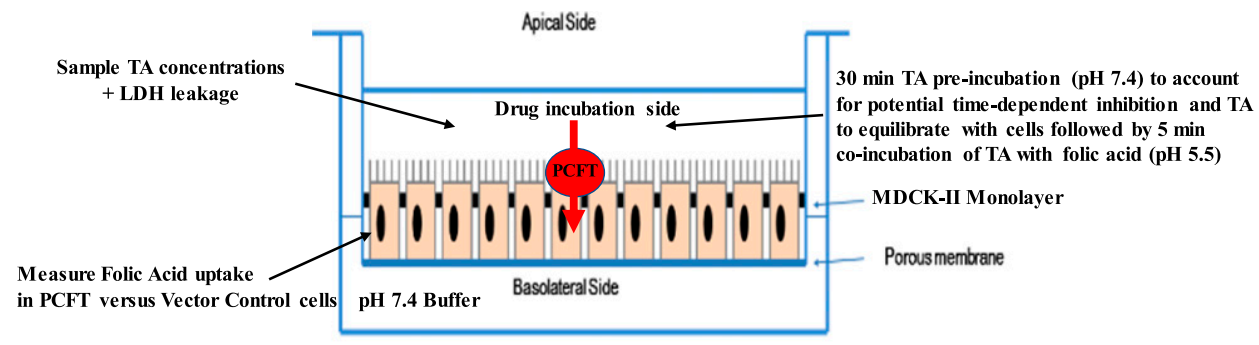

B

\section{SCHEMATIC OF RFC TRANSPORT EXPERIMENTS}

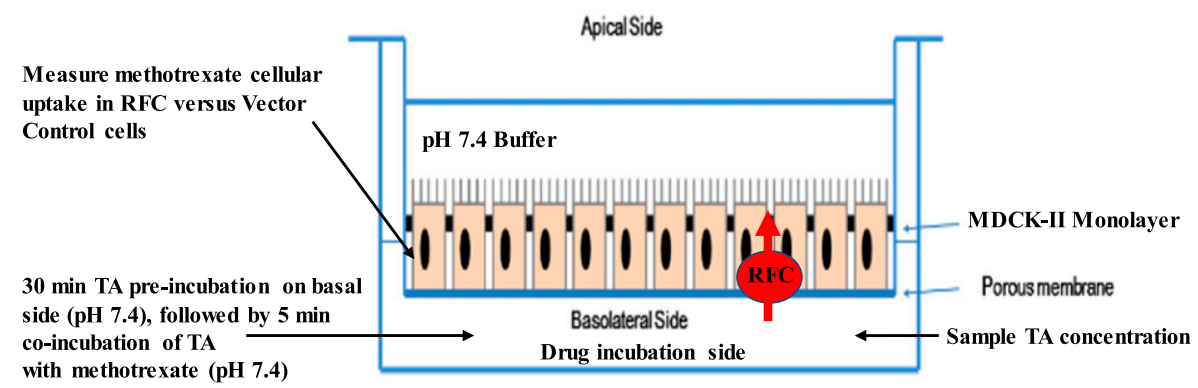

C

\section{SCHEMATIC OF FRa ENDOCYTOSIS EXPERIMENTS}

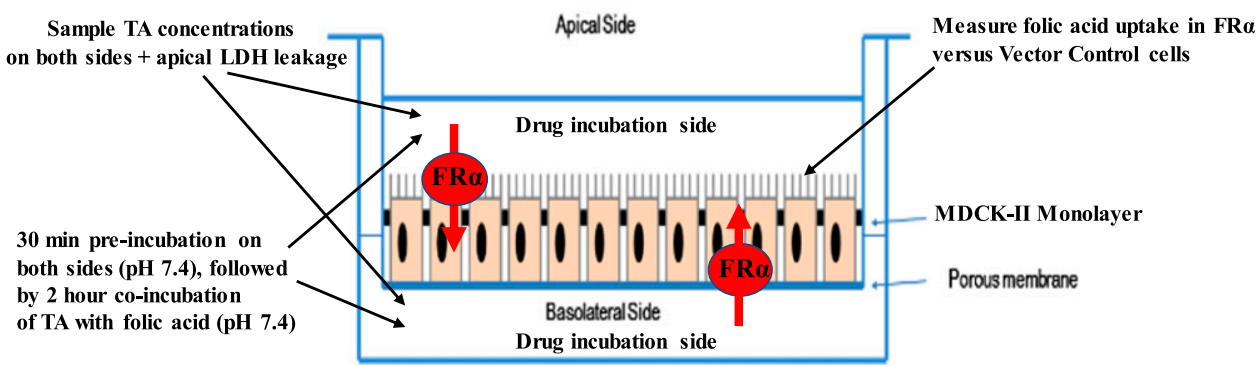

Fig. 2. Diagram of experimental setup for (A) PCFT transport, (B) RFC transport, and (C) FR $\alpha$-mediated endocytosis studies. LDH, lactate dehydrogenase; TA, test article drug. cellular uptake in RFC versus vector control cells was determined. Samples for test article concentration and lactate dehydrogenase were collected from the basolateral side.

FR $\alpha$ is expressed on both the apical and basolateral membranes of the MDCK-II renal cell line (Kim et al., 2004). As such, test articles were incubated on both apical and basolateral sides (see Fig. 2C). Preincubation with the test article ( 30 minutes) was followed by a 2-hour coincubation of $50 \mathrm{nM}\left[{ }^{3} \mathrm{H}\right]$-folic acid with the test article at $\mathrm{pH}$ 7.4. Folic acid cellular uptake in $\mathrm{FR} \alpha$ cells versus the vector control cells was determined. Samples for test article concentration were collected from both basolateral and apical sides; lactate dehydrogenase leakage was measured only on the apical side.

Specific transport activity was determined by the subtraction of the mean cellular uptake in vector control cells (incubated at the same time under same conditions) from that observed in PCFT, RFC, or FR $\alpha$ overexpressing cells ( $n=4$ wells/cell type per condition). Nominal test drug concentration ranges started at the highest soluble concentration based on visual inspection and were decreased in half-log increments for a total of eight concentrations: $0.03-100 \mu \mathrm{M}$ for dolutegravir, cabotegravir, elvitegravir and $0.3-1000 \mu \mathrm{M}$ for bictegravir, raltegravir, methotrexate, pemetrexed, and valproic acid. Actual test drug concentrations at the end of the experiment were measured and used for determination of inhibitory concentrations (see Sample Analysis and Data Analysis sections).
The following criteria were used to deem transport or endocytosis activity acceptable in any assay run: 1) PCFT-mediated folic acid uptake, $\geq 31.6 \mathrm{fmol} / \mathrm{min} / \mathrm{cm}^{2}$; RFC-mediated methotrexate uptake, $\geq 46.3 \mathrm{fmol} /$ $\min / \mathrm{cm}^{2}$; FR $\alpha$-mediated folic acid endocytosis, $\geq 4.51 \mathrm{fmol} / \mathrm{min} / \mathrm{cm}^{2}$; and 2) $\geq 70 \%$ inhibition of transporter-mediated uptake or receptormediated endocytosis by prototypical inhibitors: $300 \mu \mathrm{M}$ bromosulfophthalein for PCFT-mediated folic acid uptake, $1000 \mu \mathrm{M}$ pemetrexed for RFC-mediated methotrexate uptake, and $300 \mu \mathrm{M}$ pemetrexed for FR $\alpha$ mediated folic acid endocytosis.

\section{Sample Analysis}

The transport or endocytosis of each assay substrate was determined by radiometric detection. In the transport and endocytosis studies, the concentrations of test articles from the donor sides at the end of coincubation were measured by liquid chromatography-tandem mass spectrometry (see Supplemental Material, Method 1 for summary of liquid chromatography-tandem mass spectrometry methods). These measured concentrations were used in the calculation of all inhibitory concentrations (except second positive control, pemetrexed, for which nominal concentrations were used). Potential cytotoxicity was evaluated by the release of lactate dehydrogenase, whose activity was quantified by spectrophotometric quantification $(\lambda=590 \mathrm{~nm})$ of formazan formation using a commercial kit (G-Biosciences, St. Louis, MO). 



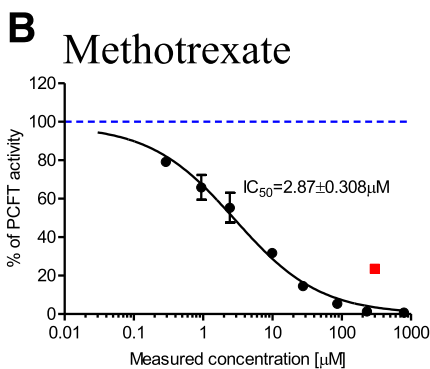

F Bictegravir

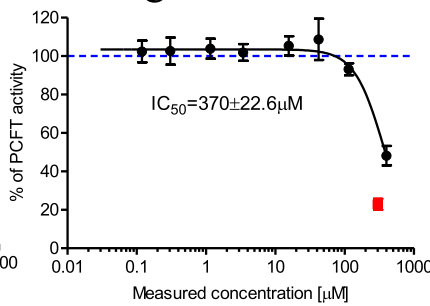

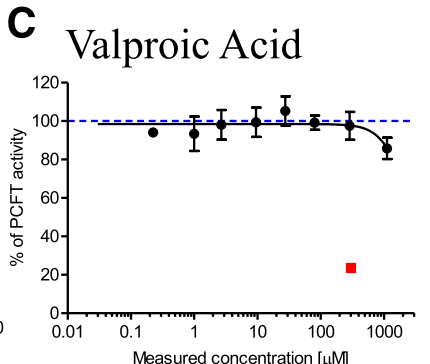

G

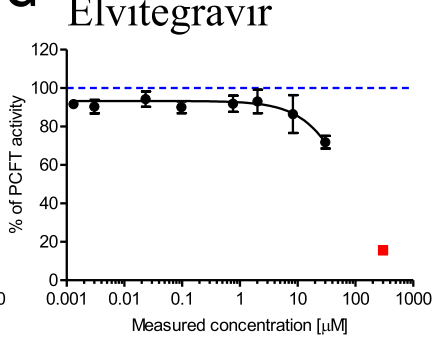

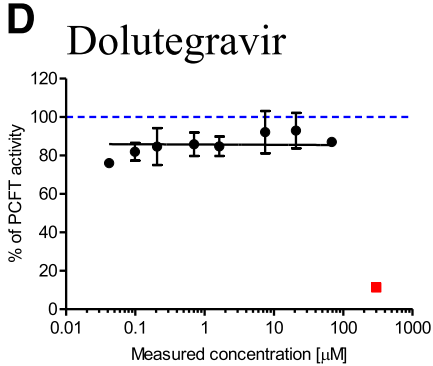



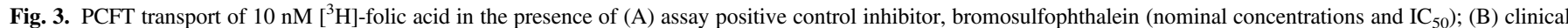

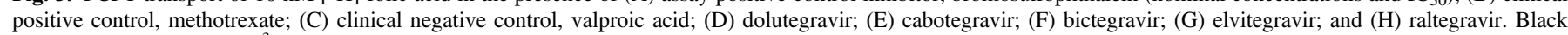

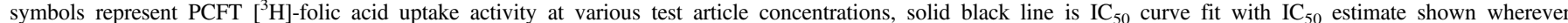

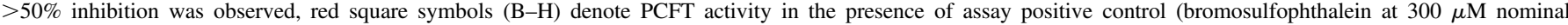


experiment, except bromosulfophthalein concentrations are nominal. Mean \pm S.D., $n=4$.

\section{Data Analysis (eq. 1-4)}

\section{In Vitro Data Analysis.}

Net transporter or receptor-mediated uptake activity $\left(\mathrm{fmol} / \mathrm{min} / \mathrm{cm}^{2}\right)$

$=[($ accumulation in overexpressing cells $)$-(accumulation in control cells $)] /$ [substrate incubation time*transwell insert surface area]

Percent transporter or receptor-mediated uptake activity

$$
\begin{aligned}
= & 100-[100 *(\text { net uptake activity } \\
& (\text { net uptake inhibitor }) / \\
& \text { nptake activity } \left.\left._{\text {without inhibitor }}\right)\right]
\end{aligned}
$$

Inhibitory concentration (IC)

Inhibitory concentration $50\left(\mathrm{IC}_{50}\right)$ values were estimated by nonlinear leastsquares fitting of the following equation to the uptake data (Prism v.5; GraphPad, San Diego, CA):

$$
\begin{aligned}
& \text { uptake activity } \text { inhibitor concentration }=\text { uptake activity }_{\text {no inhibitor }} / \\
& {\left[1+([\text { Inhibitor Concentration }] / \text { IC })^{\mathrm{n}}\right]}
\end{aligned}
$$

where $n$ is the Hill coefficient. Wherever $\geq 50 \%$ inhibition was achieved, the associated inhibitory concentration $\left(\mathrm{IC}_{50}\right)$ values are reported. Otherwise, the greatest extent of inhibition observed $\geq 25 \%$ is reported and discussed. This $\geq 25 \%$ inhibition level was selected on the basis that $\geq 25 \%$ cumulative inhibition of folate absorption, distribution, and renal tubular reabsorption (sparing) would decrease fetal folate levels >2-fold (Zamek-Gliszczynski et al., 2009, 2013).

$$
\begin{aligned}
\text { Percent cytototoxicity }= & \left(\mathrm{LDH}_{\text {inhibitor concentration }}-\mathrm{LDH}_{\text {vehicle control }}\right) / \\
& \left(\mathrm{LDH}_{1 \% \text { Tritox } \mathrm{x}}-\mathrm{LDH}_{\text {vehicle control }}\right) * 100 \%,
\end{aligned}
$$

where $\mathrm{LDH}=$ lactate dehydrogenase. Lactate dehydrogenase activity at any given inhibitor concentration had to be statistically significant ( $t$ test with Bonferroni correction) versus vehicle control, and percent cytotoxicity had to be $>12.5 \%$ ( $>$ half of minimal inhibition level considered in this study) to disqualify uptake inhibition data due to confounding cytotoxicity.

\section{Clinical Extrapolation (eq. 5-8)}

Since clinical extrapolation of in vitro nutrient-drug transport inhibition is not well established, clinical extrapolation of observed in vitro inhibitory potency values was based on established thresholds (FDA, 2017). This extrapolation framework is considered the best available and considers the worst-case scenario, although it was validated for drug-drug interactions, and not drug-folate interactions with the transport pathways studied herein specifically (eq. 5-8).

Intestinal absorption:

$$
\text { Cut }- \text { off }: \text { Igut } / \text { IC } \geq 10
$$

where $\mathrm{I}_{\text {gut }}=$ highest clinical dose $/ 250 \mathrm{ml}$ or maximal aqueous solubility and IC $=$ inhibitory concentration eliciting noted percentage of inhibition $(50 \%$ or $\geq 25 \%$ for the purpose of this study).

Distribution:

$$
\text { Cut }- \text { off }: 1+C \max , \mathbf{u} / \mathrm{IC} \geq \mathbf{1 . 1}
$$

Proximal tubule reabsoprtion (apical proximal tubule transport pathways: PCFT and $\mathrm{FR} \alpha$ ):

$$
\text { Cut }- \text { off }: \mathbf{1}+\mathrm{Cmax}, \mathrm{u} / \mathrm{IC} \geq \mathbf{1 . 0 2}
$$

Proximal tubule reabsoprtion (basolateral proximal tubule transporter: RFC):

$$
\text { Cut }- \text { off }: 1+\mathrm{Cmax}, \mathrm{u} / \mathrm{IC} \geq \mathbf{1 . 1}
$$

where $\mathrm{Cmax}, \mathrm{u}=$ unbound plasma $\mathrm{Cmax}$ at highest clinical dose; $\mathrm{IC}=$ inhibitory concentration eliciting noted percentage of inhibition $(50 \%$ or $\geq 25 \%$ for the purpose of this study).

\section{Data Presentation}

All data are reported as mean \pm S.D. $(n=4$ replicates), unless otherwise noted. Statistical significance was assessed by student's $t$ test with Bonferroni correction for multiple comparisons. All reported inhibitory concentrations refer to experimentally determined concentrations measured at the end of the coincubation period in the MDCK-II assays (except assay positive controls, bromosulfophthalein or pemetrexed, for which nominal concentrations were used). 
A Positive Control

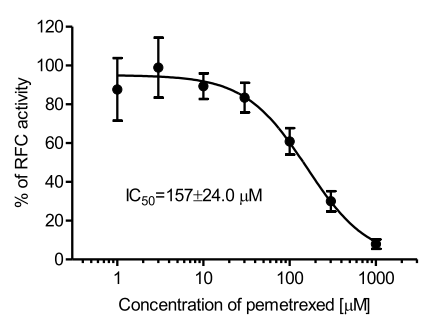

E
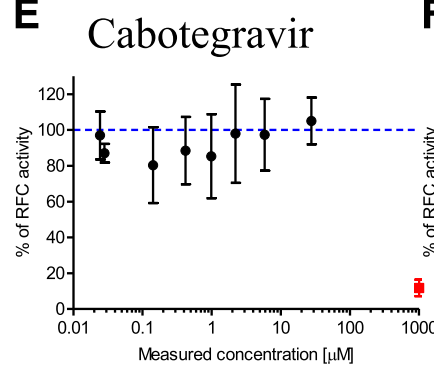

\section{B Methotrexate}

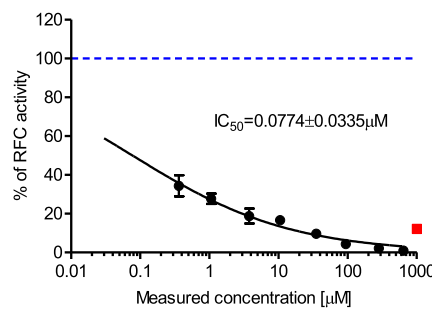

F Bictegravir

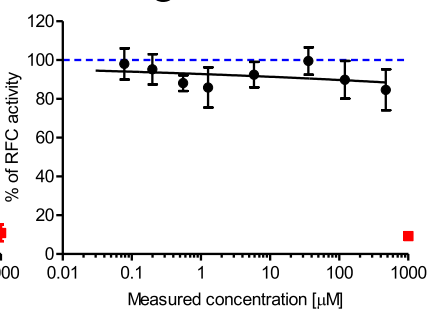

C Valproic Acid

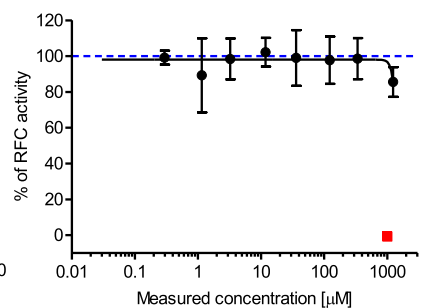

G Elvitegravir

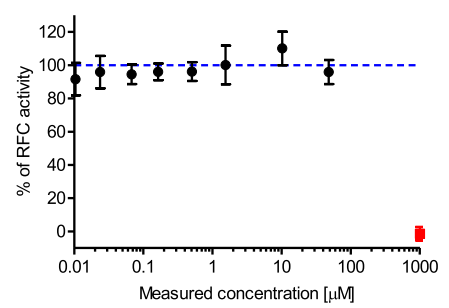

D Dolutegravir

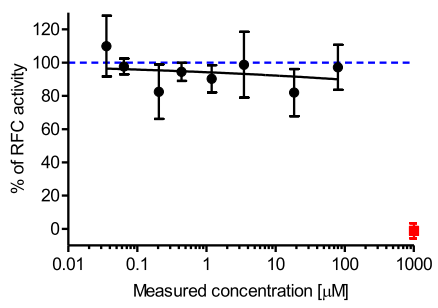

H Raltegravir

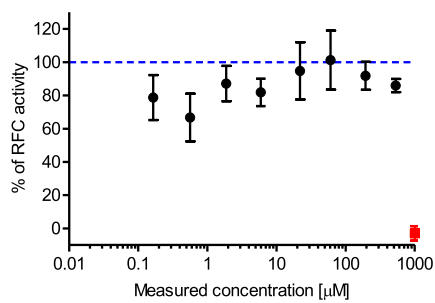

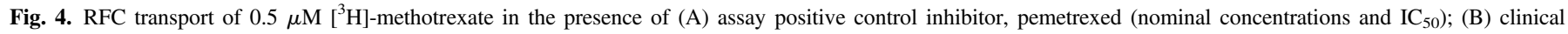

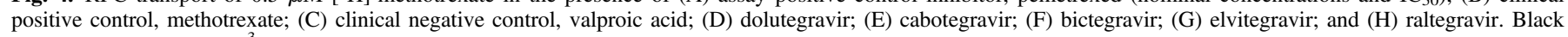



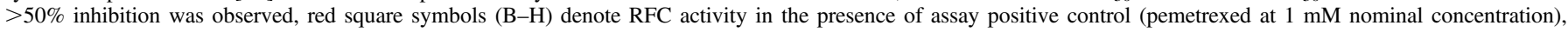

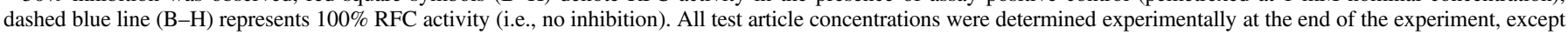
pemetrexed concentrations are nominal. Mean \pm S.D., $n=4$.

\section{Results}

Functional verification of optimized folate in vitro transport assays (Fig. 2) is summarized in Supplemental Fig. 2. Briefly, PCFT functional activity was confirmed with folic acid uptake that was, on average, 31.7fold (CV $=18.3 \%, n=3$ independent experiments) enhanced in PCFT versus vector control cells. RFC did not transport unreduced folic acid in this experimental system (data not shown), consistent with the literature (Milstein et al., 2001). Instead, methotrexate was selected as a reduced form of folic acid and demonstrated 2.1-fold ( $\mathrm{CV}=21.7 \%, n=3$ independent experiments) enhanced cellular uptake in RFC versus vector control cells. This relatively lower magnitude of RFC functional enhancement in overexpressing cells has been reported previously (Kneuer et al., 2005) and emphasizes the importance of vector-control cell activity correction to determine overexpressed RFC activity. FR $\alpha$ mediated folic acid endocytosis was 10.7 -fold $(\mathrm{CV}=16.9 \%, n=3$ independent experiments) enhanced in $\mathrm{FR} \alpha$ overexpressing versus vector control cells $\left(1.2-1.3 \times 10^{5} \mathrm{FR} \alpha \mathrm{mRNA}\right.$ overexpression was confirmed by polymerase chain reaction; data not shown). Prototypical inhibitors, bromosulfophthalein for PCFT (Nakai et al., 2007) and pemetrexed for RFC and FR $\alpha$ (Chattopadhyay et al., 2007), inhibited these respective folate transport pathways in a concentration-dependent manner $\left(\mathrm{IC}_{50}=78.1 \pm 7.5,157 \pm 24\right.$, and $17.5 \pm 1.4 \mu \mathrm{M}$ for PCFT, $\mathrm{RFC}$, and $\mathrm{FR} \alpha$, respectively), with nearly complete inhibition at the highest $1 \mathrm{mM}$ inhibitor concentration (Fig. 3-5).

PCFT inhibition results are summarized in Fig. 3. The clinical positive control, methotrexate, inhibited PCFT $\left(\mathrm{IC}_{50}=2.9 \pm 0.3 \mu \mathrm{M}\right)$; in contrast, no inhibition was observed for the clinical negative control, valproic acid (Fig. 3, B and C). Dolutegravir and cabotegravir at all test concentrations (up to maximum soluble in assay buffer) did not inhibit PCFT (Fig. 3, D and E). Bictegravir inhibited PCFT $\left(\mathrm{IC}_{50}=370 \pm\right.$ $23 \mu \mathrm{M}$; Fig. 3F). Elvitegravir elicited $28.1 \% \pm 3.3 \%$ PCFT inhibition at $30.0 \pm 2.0 \mu \mathrm{M}$, and raltegravir inhibited PCFT $32.4 \% \pm 2.4 \%$ at $564 \pm 38 \mu \mathrm{M}$ (Fig. 3, G and H).

RFC inhibition results are summarized in Fig. 4. The clinical positive control, methotrexate, inhibited $\mathrm{RFC}\left(\mathrm{IC}_{50}=0.08 \pm 0.03 \mu \mathrm{M}\right)$; no inhibition was noted for the clinical negative control, valproic acid (Fig. 4, B and C). The five integrase inhibitors drugs did not inhibit RFC transport (Fig. 4, D-H) at any concentration tested, up to the maximum soluble in assay buffer.

Inhibition of $\mathrm{FR} \alpha$-mediated folic acid endocytosis is summarized in Fig. 5. The clinical positive control, methotrexate, inhibited $\mathrm{FR} \alpha\left(\mathrm{IC}_{50}\right.$ $=50.4 \pm 5.3 \mu \mathrm{M})$; inhibition exceeding the $25 \%$ predefined threshold for this study was not observed for the clinical negative control, valproic acid (Fig. 5, B and C). Dolutegravir inhibited FR $\alpha 36.0 \% \pm 5.7 \%$ at 37.3 $\pm 4.2 \mu \mathrm{M}$, and cabotegravir inhibited $\mathrm{FR} \alpha 36.7 \% \pm 5.8 \%$ at $25.8 \pm$ $5.0 \mu \mathrm{M}$ (Fig. 5, D and E). Bictegravir inhibited folic acid FR $\alpha$-mediated endocytosis with an $\mathrm{IC}_{50}$ of $268 \pm 66 \mu \mathrm{M}$ (Fig. 5F). Although elvitegravir elicited an apparent $14 \%-38 \%$ decrease in FR $\alpha$-mediated folic acid cellular accumulation at the top two concentrations tested, this decrease was comparable in magnitude to $14 \%-27 \%$ cytotoxicity observed at these elvitegravir concentrations in this assay (Supplemental Table 3); therefore, no apparent inhibition of FR $\alpha$ by elvitegravir was concluded. Raltegravir did not inhibit FR $\alpha$ (Fig. 4H).

Drug parameters necessary for clinical extrapolation of in vitro folate transport inhibition results are presented in Table 1. Clinical extrapolations of PCFT, RFC, and $\mathrm{FR} \alpha$ inhibition in folate intestinal absorption, distribution, and renal tubular reabsorption are summarized in Fig. 6. Extrapolations for folate intestinal absorption were made only for PCFT because the other two pathways are not involved in this process, whereas all three pathways are involved in folate distribution and renal sparing (Fig. 1).

The antifolate drugs, methotrexate and pemetrexed, were flagged as clinically relevant inhibitors of PCFT, RFC, and FR $\alpha$ in folate absorption, distribution, and renal tubular reabsorption (Fig. 6; FDA, 2017). Inhibition of PCFT transport of folic acid by bictegravir, raltegravir, and elvitegravir was flagged only as potentially clinically relevant for 1200-mg oral raltegravir in intestinal folate absorption (Igut/ $\mathrm{IC}_{32}>10$ ). Otherwise, $400 \mathrm{mg}$ oral raltegravir did not meet the criteria for clinically relevant intestinal PCFT inhibition; bictegravir, raltegravir, and elvitegravir were not clinically relevant systemic PCFT inhibitors in 
A Positive Control
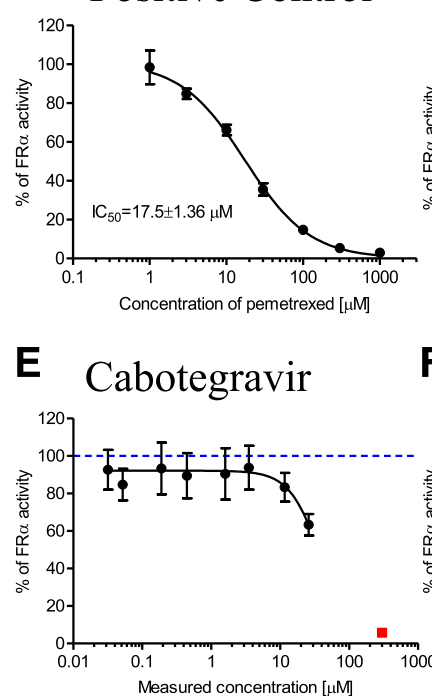

\section{B Methotrexate}

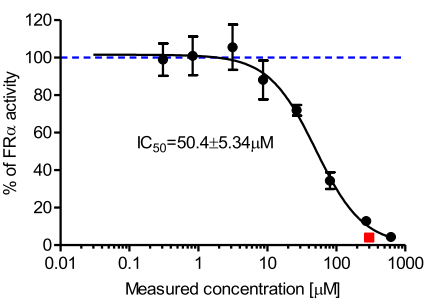

F Bictegravir

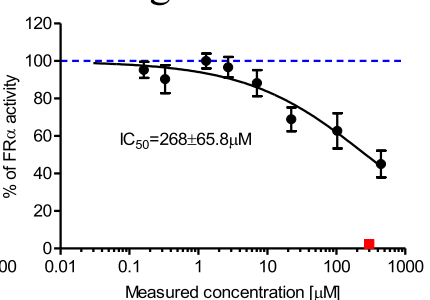

C Valproic Acid

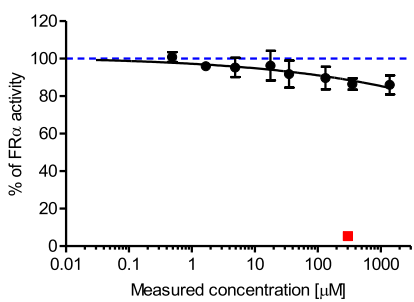

G Elvitegravir

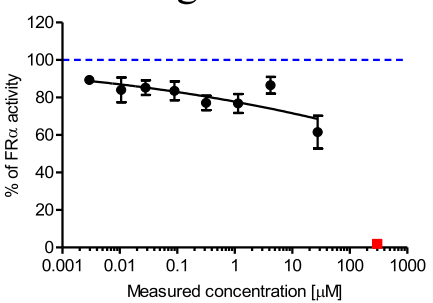

D Dolutegravir

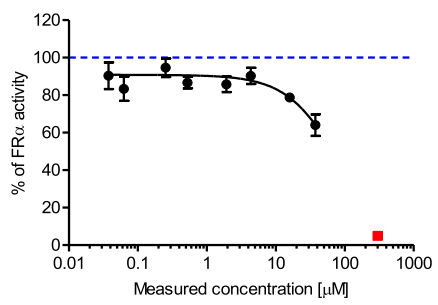

H Raltegravir

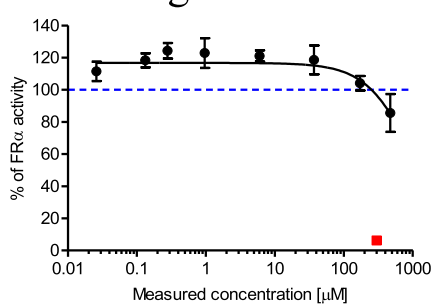

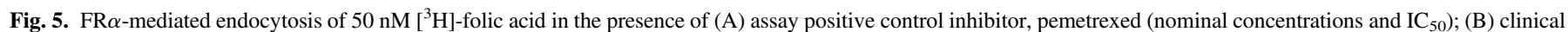

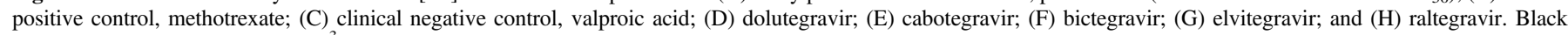

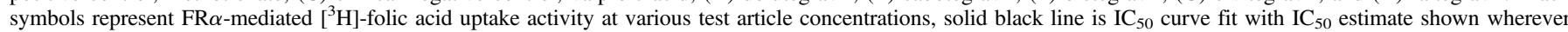

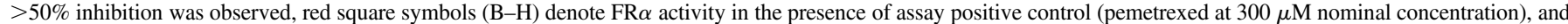

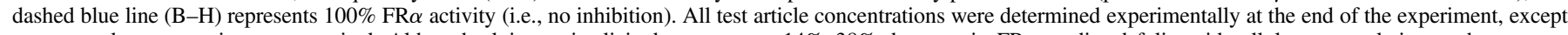

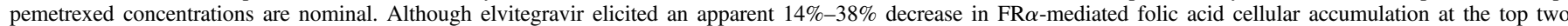


Table 3); therefore, no apparent inhibition of FR $\alpha$ by elvitegravir was concluded. Mean \pm S.D., $n=4$.

folate distribution and renal tubular reabsortion (Fig. 6; FDA, 2017). None of the five HIV integrase inhibitor drugs were considered clinical inhibitors of RFC because in vitro RFC inhibition was not observed up to their respective maximal soluble concentrations, which were sufficiently high to rule out intestinal and systemic inhibition at clinical doses and exposures (Table 1). Inhibition of FR $\alpha$-mediated folic endocytosis by dolutegravir, cabotegravir, and bictegravir observed in vitro was well below the thresholds for potential clinical relevance of transport pathways at the level of tissue distribution $\left(1+\mathrm{C}_{\max }, \mathrm{u} / \mathrm{IC}<<\right.$ 1.1) and renal tubular reabsorption on apical membrane of renal proximal tubules $(1+\mathrm{Cmax}, \mathrm{u} / \mathrm{IC}<<1.02)$ (Fig. 6; FDA, 2017).

\section{Discussion}

Preliminary analysis of an ongoing clinical birth surveillance study suggested increased risk of NTDs in newborns from mothers taking dolutegravir at the time of conception (Zash et al., 2018). Dolutegravir is an integrase inhibitor for the treatment of HIV, a drug class that also includes cabotegravir, bictegravir, raltegravir, and elvitegravir (Han et al., 2017). Folate deficiency increases the incidence rate of NTDs (Daly et al., 1995; Botto et al., 1999; Crider et al., 2014; Kancherla et al., 2018). Folate relies on transport via PCFT for intestinal absorption, FR $\alpha$-mediated endocytosis along with transport by PCFT and RFC for tissue distribution, including to the fetus, and renal sparing via active tubular reabsorption after extensive glomerular filtration (Fig. 1, Solanky et al., 2010; Zhao et al., 2011).

The aim of the present work was to determine whether dolutegravir and other integrase inhibitor drugs may be clinically relevant inhibitors of the three major folate transport pathways, which would support an increased likelihood of folate deficiency leading to increased NTD risk. Of the five HIV integrase inhibitor drugs evaluated for inhibition of PCFT, RFC, and FR $\alpha$ in folate absorption, distribution, and renal tubular reabsorption, only raltegravir at the highest (i.e., $1.2 \mathrm{~g}$ ) clinical dose was flagged as a potential clinical inhibitor of PCFT intestinal folate absorption. Otherwise, these five HIV integrase inhibitor drugs are not predicted to inhibit the three major folate transport pathways at clinical therapeutic doses and concentrations (Fig. 6; FDA, 2017).

Based on the current results, dolutegravir is not predicted to elicit clinical decreases in maternal and fetal folate levels. Furthermore, dolutegravir is not an inhibitor of DHFR, the first step in folic acid metabolism in the synthesis of nucleic and amino acids (Cabrera et al., 2019). Taken together, these results do not support dolutegravir-induced folate deficiency as a mechanistic explanation for the reported preliminary observations of increased NTD risk (Zash et al., 2018). As such, these studies do not lend mechanistic support for folic acid supplementation to overcome this proposed increased NTD risk (Zash et al., 2018); however, there is no harm to folic acid supplementation, and it is an established good practice known to reduce the risk of NTDs in the general population who may not otherwise consume adequate amounts of dietary folic acid (Botto et al., 1999; Kancherla et al., 2018). Although studies have repeatedly failed to demonstrate folic acid supplementation to reduce the 4-fold increased NTD rate in epileptic women on valproic acid treatment, folate supplementation is still recommended for women of childbearing potential taking valproic acid as a safe precaution supported by evidence in the general population ((Depakene Prescribing Information, 2011; Hansen et al., 1995; Craig et al., 1999; Candito et al., 2007; 2011)).

HIV integrase inhibitor drug class effect on folate transport pathways was not observed in the present study (Fig. 6), which is conceptually consistent with the lack of clinical evidence for increased NTD risk by this class of drugs ((Viteka Prescribing Information, 2015, Isentress (raltegravir), 2019, Stribild Prescribing Information (2019))). Even raltegravir, which was flagged as a potentially clinically relevant intestinal PCFT inhibitor at the highest $1.2 \mathrm{~g}$ clinical dose is not known to increase the clinical risk of NTDs or cause birth defects in preclinical embryofetal toxicology studies (Isentress (raltegravir), 2019).

After the report of potentially increased prevalence of NTDs in the ongoing dolutegravir birth surveillance study (Zash et al., 2018), in vitro $\mathrm{FR} \alpha$ receptor binding experiments were used to demonstrate that 
TABLE 1

Drug parameters relevant for clinical extrapolation of in vitro transport inhibition

\begin{tabular}{|c|c|c|c|c|c|c|c|}
\hline Drug & Mol. Wt. & Dose & $\mathrm{C}_{\max }$ & $f_{\mathrm{u}}$ & $\mathrm{C}_{\max , \mathrm{u}}$ & $\mathrm{I}_{\text {gut }}$ & Reference \\
\hline & $\mathrm{g} / \mathrm{mol}$ & $m g$ & $\mu M$ & & $\mu M$ & $\mu M$ & \\
\hline Dolutegravir & 441.36 & 50 & $8.32^{a}$ & 0.01 & $0.08^{a}$ & 453.14 & Tivicay, Prescribing Information, 2018 \\
\hline Cabotegravir & 427.33 & 30 & 14.88 & 0.01 & 0.15 & 280.81 & Ford et al., 2019 \\
\hline Bictegravir & 449.38 & 50 & 13.69 & 0.01 & 0.14 & 445.06 & Biktarvy, Prescribing Information, 2018 \\
\hline Raltegravir & 482.51 & 400 & 4.50 & 0.17 & 0.76 & 3315.99 & Isentress (raltegravir) Prescribing Information, 2019 \\
\hline Raltegravir & 482.51 & 1200 & 22.56 & 0.17 & 3.84 & 9947.98 & http://wwwcroiconferenceorg/sessions/multiple-dose-study-raltegravir-ral-formulations \\
\hline Elvitegravir & 447.88 & 85 & $2.68^{b}$ & 0.02 & $0.05^{b}$ & 759.13 & Viteka Prescribing Information, 2015 \\
\hline Elvitegravir & 447.88 & 150 & $3.35^{b}$ & 0.02 & $0.07^{b}$ & 1339.64 & Viteka Prescribing Information, 2015 \\
\hline Methotrexate & 454.44 & 10 & 0.57 & 0.5 & 0.28 & 88.02 & Jylamvo (methotrexate) Prescribing Information, 2017 \\
\hline Methotrexate (i.v.) & 454.44 & 15 & 3.80 & 0.5 & 1.90 & N/A & Seideman et al., 1993 \\
\hline Pemetrexed (i.v.) & 597.49 & $500 \mathrm{mg} / \mathrm{m}^{2}$ & 189.12 & 0.19 & 35.93 & N/A & Kavathiya et al., 2017 \\
\hline
\end{tabular}

$C_{\max , \mathrm{u}}$ unbound $C_{\max }$ (i.e., $C_{\max } * f_{\mathrm{u}}$ ); $f_{\mathrm{u}}$, plasma fraction unbound; $I_{\mathrm{gu}}$, theoretical gut concentration (oral dose/250 ml) or highest soluble aqueous concentration; oral administration in all cases, unless noted); N/A, not applicable (i.v. administration).

${ }^{a}$ Most commonly used 50-mg daily regimen; $50 \mathrm{mg}$ twice daily; $C_{\max }=9.4 \mu \mathrm{M}$ and $C_{\max , \mathrm{u}}=0.09 \mu \mathrm{M}$ (ViiV Healthcare, 2018), twice-daily dosing does not impact extrapolation values or results in Fig. 6.

${ }^{b}$ Not cobicistat boosted, 150-mg dose cobicistat-boosted $C_{\max }=3.8 \mu \mathrm{M}$ and $C_{\max , \mathrm{u}}=0.08 \mu \mathrm{M}$ (2019); cobicistat boosting does not impact extrapolation values or results in Fig. 6 .

dolutegravir is a noncompetitive FR $\alpha$ antagonist (Cabrera et al., 2019). Dolutegravir inhibited folic acid binding to $\mathrm{FR} \alpha$ up to $44 \%$ at concentrations between 16 and $64 \mu \mathrm{M}$, with an apparent $\mathrm{IC}_{50}$ of $4.4 \mu \mathrm{M}$, where $\mathrm{IC}_{50}$ was defined as dolutegravir concentration eliciting $22 \%$ inhibition of folic acid binding to $\operatorname{FR} \alpha$ (i.e., half the maximal inhibitory effect) (Cabrera et al., 2019). Considering that Cabrera et al. examined receptor binding as opposed to functional receptor-mediated endocytosis reported in this study, the dolutegravir $\operatorname{FR} \alpha$ antagonism results (44\% inhibition at dolutegravir concentrations 16-64 $\mu \mathrm{M}$ ) are in good agreement with $36 \%$ inhibition of $\mathrm{FR} \alpha$-mediated folic acid at $37 \mu \mathrm{M}$ dolutegravir (Fig. 5D; Fig. 6); however, Cabrera et al. (2019) concluded that dolutegravir's apparent $\mathrm{FR} \alpha$ inhibitory potency of $4.4 \mu \mathrm{M}$ occurred at therapeutic concentrations based on direct comparison with clinical total plasma concentrations (Table 1; Tivicay, Prescribing Information, 2018). Dolutegravir is highly plasma protein bound ( $\geq 98.9 \%$; Table 1; Tivicay, Prescribing Information, 2018), and because only unbound drug is available for pharmacologic activity (i.e., on- and off-target binding, interactions with transporters and drugmetabolizing enzymes, tissue distribution, etc.), direct comparison of in vitro potencies to total plasma concentrations is not clinically pertinent (Smith et al., 2010). Such a direct in vitro to total plasma comparison may have been possible if $4 \%$ albumin were added to the buffer in the FR $\alpha$-binding study to mimic plasma protein binding, but the comparison was made directly between in vitro potency in a protein-free buffer and clinical total plasma concentrations for a highly bound drug (Cabrera et al., 2019), where clinical unbound plasma concentrations are two orders of magnitude lower than total concentrations (Table 1; Tivicay, Prescribing Information, 2018).

Using the accepted clinical extrapolation approach for the dolutegravir apparent $\mathrm{FR} \alpha$ inhibitory potency of $4.4 \mu \mathrm{M}$ in the receptor binding assay, $1+\mathrm{C}_{\max }, \mathrm{u} / \mathrm{IC}_{50}=1.017$ (Table 1; FDA, 2017; Cabrera et al., 2019), which is not flagged as clinically relevant at the level of tissue distribution $\left(1+\mathrm{C}_{\max }, \mathrm{u} / \mathrm{IC}<1.1\right)$ and renal sparing on apical membrane of renal proximal tubules $\left(1+\mathrm{C}_{\max }, \mathrm{u} / \mathrm{IC}<1.02\right)$ (FDA, 2017). Since $50 \% \mathrm{FR} \alpha$ antagonism was not achieved, the apparent $\mathrm{IC}_{50}$ is really an $\mathrm{IC}_{22}$ (Cabrera et al., 2019), and it is not accepted practice to use this apparent $\mathrm{IC}_{50}$ parameter in clinical extrapolations (FDA, 2017). Applying the present extrapolation approach to inhibition of folate transport pathways, where the greatest inhibitory potency $\geq 25 \%$ is used wherever $50 \%$ inhibition was not achieved, $\mathrm{FR} \alpha$ antagonism $\mathrm{IC}_{44}=16 \mu \mathrm{M}$ (Cabrera et al., 2019), such that $1+\mathrm{C}_{\max }, \mathrm{u} / \mathrm{IC}_{50}=1.005$ and well below thresholds for potential clinical significance (Table 1; FDA, 2017; Cabrera et al., 2019).

In the present study, clinical extrapolation of observed in vitro inhibitory potency values were based on established thresholds for clinically relevant transporter inhibition in the intestine $\left(\mathrm{I}_{\mathrm{gut}} / \mathrm{IC}_{50} \geq 10\right)$, systemically $\left(1+\mathrm{C}_{\max }, \mathrm{u} / \mathrm{IC}_{50} \geq 1.1\right)$, and on the apical membrane of renal proximal tubules $\left(1+\mathrm{C}_{\max }, \mathrm{u} / \mathrm{IC}_{50} \geq 1.02\right)$ (FDA, 2017). Although it was validated for transporter-based drug-drug interactions, the principles should apply to drug-nutrient interactions. This extrapolation framework is considered the best available and a conservative approach. As theoretically expected, intensive study of clinical translation of in vitro transport pathway inhibition demonstrated that the same translational pharmacokinetic principles apply to different transporter processes located at the same site in the body (i.e., the same principles and thresholds apply to different transporters localized in the intestine, liver, and basolateral or apical membrane of renal proximal tubules) (Giacomini et al., 2010), Hillgren et al., 2013; FDA, 2017; ZamekGliszczynski et al., 2018). Furthermore, these translational approaches have precedent in the extrapolation of in vitro intestinal and systemic thiamine transport by drugs as an alert to drug-induced Wernicke's disease (Giacomini et al., 2017). Notably, these approaches are based on $\mathrm{IC}_{50}$ values where $>50 \%$ transport function impairment was observed in vitro, and in the present study any inhibitor concentration associated with the greatest extent of inhibition observed $\geq 25 \%$ was reported and extrapolated (Fig. 6). This $\geq 25 \%$ inhibition level was selected on the basis that $\geq 25 \%$ of the cumulative inhibition of folate absorption, distribution, and renal tubular reabsorption (sparing) would decrease fetal folate levels $>2$-fold (Zamek-Gliszczynski et al., 2009, 2013). The present approach of extrapolating any noted in vitro inhibition $\geq 25 \%$ of folate transport pathways was deliberately conservative and intended to flag any potential clinical inhibition by the five integrase inhibitor drugs. Even with this conservative $\geq 25 \%$ inhibition approach, the present studies do not support 1) dolutegravir as a clinical inhibitor of the three major folate transport pathways or 2) clinically relevant effect of the integrase inhibitor drug class on folate transport.

The present studies are limited to acute inhibition effects by parent drugs. Parent dolutegravir is the predominant circulating moiety with negligible systemic metabolite exposure (Moss et al., 2015). As such, total radioactivity can be assumed to approximate parent drug concentrations, and relevant tissue-to-blood ratios can be estimated from wholebody autoradiography studies in pregnant rats (unpublished data GlaxoSmithKline study 2012N137348_00): placenta-to-blood ratio of $0.59 \pm 0.10$, fetal-to-maternal blood ratio of $0.13 \pm 0.02$, and kidney-toblood ratio of $0.42 \pm 0.03$. The clinical translational framework based on unbound systemic drug levels is supported by these tissue-to-blood ratios, which do not indicate preferential fetal or renal drug partitioning, as well as systemic exposure primarily to parent dolutegravir and not 


\begin{tabular}{|c|c|c|c|c|c|c|c|}
\hline \multirow{3}{*}{ Drug } & \multicolumn{7}{|c|}{ Inhibition ${ }^{*}\left(\mathrm{IC}_{50}\right.$ or $\left.\mathrm{IC}_{225 \%}\right)$} \\
\hline & \multicolumn{3}{|c|}{ PCFT } & \multicolumn{2}{|c|}{ RFC } & \multicolumn{2}{|c|}{ FR $\alpha$} \\
\hline & Absorption & Distribution & Renal sparing & Distribution & Renal sparing & Distribution & Renal sparing \\
\hline dolutegravir & & & & & & 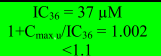 & 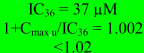 \\
\hline cabotegravir & & & & & & 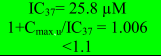 & $\begin{array}{c}\mathrm{IC}_{33}=25.8 \mu \mathrm{M} \\
1+\mathrm{C}_{\max } / \mathrm{II}_{37} \\
<1.02\end{array}$ \\
\hline bictegravir & 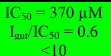 & $\begin{array}{l}\mathrm{IC}_{50}=370 \mu \mathrm{M} \\
1+\mathrm{C}_{\max } / / \mathrm{I}_{50}=1.0004 \\
<1.1\end{array}$ & $\begin{array}{l}\mathrm{IC}_{50}=370 \mu \mathrm{M} \\
1+\mathrm{C}_{\max } / \mathrm{I} \mathrm{C}_{50}= \\
1.0004<1.02\end{array}$ & & & $\begin{array}{c}\mathrm{IC}_{s=2}=268 \mu \mathrm{M} \\
1+\mathrm{C}_{\max } / \mathrm{II}_{50}=1.0005 \\
<1.1\end{array}$ & $\begin{array}{l}\mathrm{IC}_{50}=268 \mu \mathrm{MM} \\
1+\mathrm{C}_{\max } / \mathrm{I} \mathrm{C}_{50}= \\
10005<1.02\end{array}$ \\
\hline raltegravir & 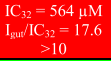 & $\begin{array}{c}\mathrm{IC}_{32}=564 \mu \mathrm{M} \\
1+C_{\max } / I_{32}=1.007 \\
<1.1 \\
\end{array}$ & 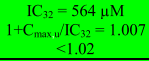 & & & & \\
\hline elvitegravir & $\begin{array}{l}\mathrm{IC}_{28}=30 \mu \mathrm{M} \\
\mathrm{I}_{\mathrm{pu} u} / \mathrm{I}_{28}=0.02 \\
<10\end{array}$ & $\begin{array}{c}\mathrm{IC}_{28}=30 \mu \mathrm{M} \\
1+\mathrm{C}_{\max } / \mathrm{JI}_{2.8}=1.002 \\
<1.1\end{array}$ & 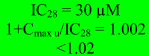 & & & & \\
\hline methotrexate & $\begin{array}{l}\mathrm{IC}_{50}=2.9 \mu \mathrm{M} \\
\mathrm{I}_{\mathrm{gu}} / \mathrm{C}_{50}=31 \\
>10\end{array}$ & $\begin{array}{c}\mathrm{IC}_{5_{5}}=2.9 \mu \mathrm{M} \\
1+\mathrm{C}_{\max , \mathrm{N} / \mathrm{I} \mathrm{S}_{50}=1.7}\end{array}$ & $\begin{array}{c}1 \mathrm{C}_{50}=2.9 \mu \mathrm{M} \\
1+\mathrm{C}_{\max ,} / \mathrm{C}_{50}=1.7 \\
>1.02\end{array}$ & $\begin{array}{c}1 \mathrm{C}_{50}=0.08 \mu \mathrm{M} \\
1+\mathrm{C}_{\max }, / \mathrm{IC}_{50}=50 \\
>1.1\end{array}$ & $\begin{array}{c}\mathrm{IC}_{\mathrm{S}_{0}}=0.08 \mu \mathrm{M} \\
1+\mathrm{C}_{\max , / / \mathrm{C}_{50}} \\
>1.1\end{array}$ & 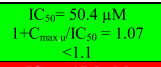 & $\begin{array}{c}\mathrm{IC}_{s}=50.4 \mu \mathrm{M} \\
1+\mathrm{C}_{\max } / \mathrm{C}_{s_{0}}=1.07 \\
>1.02\end{array}$ \\
\hline $\begin{array}{c}\text { pemetrexed } \\
\text { (nominal conc) }\end{array}$ & IV Dose - N/A & $\mathrm{IC}_{50}-\mathrm{N} / \mathrm{D}$ & $\mathrm{IC}_{50}-\mathrm{N} / \mathrm{D}$ & $\begin{array}{c}\mathrm{C}_{s_{0}}=157 \mu \mathrm{M} \\
1+\mathrm{C}_{\operatorname{mxx}} / \mathrm{d} / \mathrm{C}_{50}=1.2 \\
>1.1\end{array}$ & 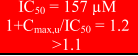 & 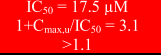 & $\begin{array}{c}\mathrm{I}_{50}=17.5 \mu \mathrm{M} \\
1+\mathrm{C}_{\max } / \mathrm{d} / \mathrm{C}_{50}=3.1 \\
>1.02\end{array}$ \\
\hline valproic acid & & & & & & $\mathrm{IC}_{15}=1380 \mu \mathrm{M}^{1}$ & $\mathrm{IC}_{15}=1380 \mu \mathrm{M}^{1}$ \\
\hline
\end{tabular}

No in vitro inhibition up to the highest soluble concentration

In vitro inhibition observed - NOT clinically relevant (FDA, 2017)

In vitro inhibition observed and POTENTIAL CLINICAL RISK OF INHIBITION (FDA, 2017)

IV dose - N/A (Not Applicable) or $\mathrm{IC}_{50} \mathrm{~N} / \mathrm{D}$ (Not Determined)

*None of the reported inhibitory data are an artifact of cytotoxicity (see Supplemental Table 3)

${ }^{1}$ Did not meet pre-defined $\geq 25 \%$ inhibition threshold for FR $\alpha$-mediated endocytosis, but a consistent trend of apparently concentration-dependent inhibition was noted up to $15 \%$ at the highest concentration tested

Cmax, $\mathbf{u}=$ unbound maximal plasma concentration, Igut $=$ theoretical gut concentration (oral dose $/ 250 \mathrm{~mL}$ ) or highest soluble aqueous concentration, IC $=$ inhibitory concentration
Fig. 6. Clinical extrapolation summary for PCFT, RFC, and FR $\alpha$ inhibition in folate absorption, distribution, and renal sparing. metabolites; however, potential effects of dolutegravir-glucuronide, the major form of dolutegravir in urine (Moss et al., 2015), on folate tubular reabsorption have not been ruled out by the present studies. Furthermore, potential regulation effects on folate transport and metabolism that may occur upon chronic dolutegravir administration remain to be investigated. These would be most relevant to study clinically in terms of blood folate, folate renal clearance, and maternal-to-neonate blood ratio, because clinical translation of in vitro folate transport and metabolism regulation is not established.

\section{Acknowledgments}

We thank Drs. Kim Adkison, Joseph Polli, Dinesh Stanislaus, and Nassrin Payvandi for their scientific input.

\section{Authorship Contributions}

Participated in research design: Zamek-Gliszczynski, Zhang, Mudunuru, Taskar, J. Huang, Y. Huang, Romach.

Conducted experiments: Zhang, Du, Chen.

Contributed new reagents and analytic tools: Zhang, Du, Chen, J. Huang, Y. Huang.

Performed data analysis: Zamek-Gliszczynski, Zhang, Mudunuru, Chen,

Taskar, Romach.

Wrote or contributed to the writing of the manuscript: Zamek-Gliszczynski, Zhang, Mudunuru, Taskar, Romach.

\section{References}

Alimta (pemetrexed). (2019) Prescribing Information. Eli Lilly and Company, Indianapolis, IN. Biktarvy (bictegravir/emtricitabine/tenofovir alafenamide). (2018) Prescribing Information. Gilead Sciences, Inc., Foster City, CA.

Botto LD, Moore CA, Khoury MJ, and Erickson JD (1999) Neural-tube defects. N Engl J Med 341: 1509-1519.

Cabrera RM, Souder JP, Steele JW, Yeo L, Tukeman G, Gorelick DA, and Finnell RH (2019) The antagonism of folate receptor by the integrase inhibitor dolutegravir Available from: https://doi. org/10.1101/576272.

Candito M, Naimi M, Boisson C, Rudigoz JC, Gaucherand P, Guéant JL, Luton D, and Van Obberghen E (2007) Plasma vitamin values and antiepileptic therapy: case reports of pregnancy outcomes affected by a neural tube defect. Birth Defects Res A Clin Mol Teratol 79:62-64.

Chattopadhyay S, Moran RG, and Goldman ID (2007) Pemetrexed: biochemical and cellular pharmacology, mechanisms, and clinical applications. Mol Cancer Ther 6:404-417.
Cohen IJ (2017) Neurotoxicity after high-dose methotrexate (MTX) is adequately explained by insufficient folinic acid rescue. Cancer Chemother Pharmacol 79:1057-1065.

Craig J, Morrison P, Morrow J, and Patterson V (1999) Failure of periconceptual folic acid to prevent a neural tube defect in the offspring of a mother taking sodium valproate. Seizure 8:253-254.

Crider KS, Devine O, Hao L, Dowling NF, Li S, Molloy AM, Li Z, Zhu J, and Berry RJ (2014) Population red blood cell folate concentrations for prevention of neural tube defects: Bayesian model. BMJ 349:g4554.

Daly LE, Kirke PN, Molloy A, Weir DG, and Scott JM (1995) Folate levels and neural tube defects: implications for prevention. JAMA 274:1698-1702.

Depakene (valproic acid). (2011) Prescribing Information. Abbott Laboratories. Chicago, IL.

DeSesso JM and Goeringer GC (1991) Amelioration by leucovorin of methotrexate developmental toxicity in rabbits. Teratology $43: 201-215$.

Food and Drug Administration (FDA) (2017) In vitro metabolism- and transporter- mediated drugdrug interaction studies (pharmacology CED, Denver CO) Office of Communications, FDA CDER, Rockville, MD

Ford SL, Lou Y, Lewis N, Kostapanos M, D'Amico R, Spreen W, and Patel P (2019) Effect of rifabutin on the pharmacokinetics of oral cabotegravir in healthy subjects. Antivir Ther DOI: 10. 3851/IMP3306 [published ahead of print].

Giacomini KM, Huang SM, Tweedie DJ, Benet LZ, Brouwer KL, Chu X, Dahlin A, Evers R, Fischer V, Hillgren KM, et al.; International Transporter Consortium (2010) Membrane transporters in drug development. Nat Rev Drug Discov 9:215-236.

Giacomini MM, Hao J, Liang X, Chandrasekhar J, Twelves J, Whitney JA, Lepist EI, and Ray AS (2017) Interaction of 2,4-diaminopyrimidine-containing drugs including fedratinib and trimethoprim with thiamine transporters. Drug Metab Dispos 45:76-85.

Han Y, Mesplède T, and Wainberg MA (2017) Investigational HIV integrase inhibitors in phase I and phase II clinical trials. Expert Opin Investig Drugs 26:1207-1213.

Hansen DK, Grafton TF, Dial SL, Gehring TA, and Siitonen PH (1995) Effect of supplementa folic acid on valproic acid-induced embryotoxicity and tissue zinc levels in vivo. Teratology $\mathbf{5 2}$ : 277-285.

Hillgren KM, Keppler D, Zur AA, Giacomini KM, Stieger B, Cass CE, and Zhang L; International Transporter Consortium (2013) Emerging transporters of clinical importance: an update from the International Transporter Consortium. Clin Pharmacol Ther 94:52-63.

Isentress (raltegravir). (2019) Prescribing Information. Merck Sharp \& Dohme Corp, Kenilworth, NJ.

Jylamvo (methotrexate). (2017) EPAR: Summary for the public. European Medicines Agency, Amsterdam.

Kancherla V, Wagh K, Johnson Q, and Oakley GP Jr (2018) A 2017 global update on folic acidpreventable spina bifida and anencephaly. Birth Defects Res 110:1139-1147.

Kavathiya K, Gurjar M, Patil A, Naik M, Noronha V, Joshi A, Gota V, and Prabhash K (2017) A comparative pharmacokinetic study of 2 pemetrexed formulations in Indian adult chemonaive patients with adenocarcinoma stage III/IV non-small cell lung cancer. Clin Pharmacol Drug Dev 6:234-239.

Kim CH, Park YS, Chung KN, and Elwood PC (2004) Sorting of the human folate receptor in MDCK cells. J Biochem Mol Biol 37:362-369.

Kneuer C, Honscha KU, and Honscha W (2005) Rat reduced-folate carrier-1 is localized basolaterally in MDCK kidney epithelial cells and contributes to the secretory transport of methotrexate and fluoresceinated methotrexate. Cell Tissue Res 320:517-524.

Matherly LH, Hou Z, and Deng Y (2007) Human reduced folate carrier: translation of basic biology to cancer etiology and therapy. Cancer Metastasis Rev 26:111-128.

Milstein MS, Kapatos G, Levine RA, and Shane B (2001) Chemisty and Biology of Pteridines and Folates, Springer US, Bethesda, MD. 
Moss L, Wagner D, Kanaoka E, Olson K, Yueh YL, and Bowers GD (2015) The comparative disposition and metabolism of dolutegravir, a potent HIV-1 integrase inhibitor, in mice, rats, and monkeys. Xenobiotica 45:60-70.

Nakai Y, Inoue K, Abe N, Hatakeyama M, Ohta KY, Otagiri M, Hayashi Y, and Yuasa H (2007) Functional characterization of human proton-coupled folate transporter/heme carrier protein 1 heterologously expressed in mammalian cells as a folate transporter. J Pharmacol Exp Ther 322:469-476.

Seideman P, Beck O, Eksborg S, and Wennberg M (1993) The pharmacokinetics of methotrexate and its 7-hydroxy metabolite in patients with rheumatoid arthritis. Br J Clin Pharmacol 35: 409-412.

Smith DA, Di L, and Kerns EH (2010) The effect of plasma protein binding on in vivo efficacy: misconceptions in drug discovery. Nat Rev Drug Discov 9:929-939.

Solanky N, Requena Jimenez A, D'Souza SW, Sibley CP, and Glazier JD (2010) Expression of folate transporters in human placenta and implications for homocysteine metabolism. Placenta 31:134-143 Stribild (elvitegravir/cobicistat/emtricitabine/tenofovir disoproxil fumarate). (2019) Prescribing Information. Gilead Sciences, Inc., Foster City, CA

Tivicay (dolutegravir). (2018) Prescribing Information. ViiV Healthcare, Research Triangle, NC Vitekta (elvitegravir). (2015) Prescribing Information. Gilead Sciences, Inc., Foster City, CA.

Zamek-Gliszczynski MJ, Kalvass JC, Pollack GM, and Brouwer KL (2009) Relationship between drug/metabolite exposure and impairment of excretory transport function. Drug Metab Dispos 37:386-390.
Zamek-Gliszczynski MJ, Lee CA, Poirier A, Bentz J, Chu X, Ellens H, Ishikawa T, Jamei M, Kalvass JC, Nagar S, et al.; International Transporter Consortium (2013) ITC recommendations for transporter kinetic parameter estimation and translational modeling of transport-mediated PK and DDIs in humans. Clin Pharmacol Ther 94 64-79.

Zamek-Gliszczynski MJ, Taub ME, Chothe PP, Chu X, Giacomini KM, Kim RB, Ray AS, Stocke SL, Unadkat JD, Wittwer MB, et al.; International Transporter Consortium (2018) Transporter in drug development: 2018 ITC recommendations for transporters of emerging clinical importance. Clin Pharmacol Ther 104:890-899.

Zash R, Makhema J, and Shapiro RL (2018) Neural-tube defects with dolutegravir treatment from the time of conception. $N$ Engl J Med 379:979-981.

Zhao R, Diop-Bove N, Visentin M, and Goldman ID (2011) Mechanisms of membrane transport of folates into cells and across epithelia. Annи Rev Nutr 31:177-201.

Address correspondence to: Dr. Maciej J. Zamek-Gliszczynski, GlaxoSmithKline, 1250 South Collegeville Rd, Collegeville, PA 19426. E-mail: maciej.x.zamekgliszczynski@gsk.com 